\title{
The influence of longitudinal wall-oscillation on the plane Poiseuille flow
}

\author{
T. Atobe
}

Japan Aerospace Exploration Agency, Japan

\begin{abstract}
Influence of wall oscillation on laminar-turbulent transition of the plane Poiseuille flow is investigated analytically and numerically. For the analytical approach, the Floquet theory using a time-dependent Orr-Sommerfeld equation is employed to estimate the fundamental aspect of this flow. The velocity profiles for the analysis are assumed as the superposition of the plane Poiseuille flow and the Stokes layer. Also the collocation method is used in the wallnormal direction for making the monodromy matrix. Depending on the parameters, namely frequency $\Omega$ and amplitude $U_{w}$ of the wall-oscillation, there is the stable region in $\Omega-U_{w}$ plane even though the Reynolds number is of supercritical. Direct numerical simulation (DNS) based on the Fourier spectral method is also employed to confirm the analytical results and to clear the flow structures. The results by DNS show good agreement with the Floquet analysis and also reveal that the vortex structures in the vicinity of the walls are strongly affected by the wall oscillation.
\end{abstract}

Keywords: plane Poiseuille flow, wall oscillation, Floquet analysis, DNS.

\section{Introduction}

The flow in the nature is basically turbulent and chaotic, and it usually doesn't have a regular structure $[1,2]$. However, it is well known that the oscillating flow or pulsatile flow show some interesting features. For example, the blood flow in arteries demonstrate the drag reduction [3]. For the oscillating flow, the plane Poiseuille flow with spanwise wall oscillation also shows the drag reduction [4] and Quadrio and Ricco [5] quantity estimated more than 40\%. It is thought that this phenomenon is concerned with the existence of the streak structures $[6,7]$. 
On the other hand, the plane Poiseuille flow with longitudinal wall oscillation is also investigated by many researchers because this flow is basically twodimensional and it seems that the analytical approach is more suitable [8-12]. Most general way to deal this flow is to assume as a combination of the plane Poiseuille flow with the Stokes layer. Some previous study investigated this modified flow and discussed the characteristics of the stability. However, in contrast to the flow with spanwise oscillation, the numerical studies are not many. Thus the present study aims to analyse the flow with spanwise oscillation by direct numerical simulation (DNS) comparing with the linear stability analysis.

In section 2, the modified flow and the parameters are shown. In section 3, the basic idea of linear stability analysis is explained. In the present study, the Floquet method is employed because of periodicity of the modified flow. The collocation method is also used to construct the mathematical formulation. The numerical scheme of DNS is given in section 4. The results obtained from both approaches are shown in section 5 and the conclusion is given in section 6 .

\section{Modified flow}

In the present study, the flow dealt here is described by a linear superposition of the plane Poiseuille flow with the Stokes layer. The distance between the walls is $2 h$, and the amplitude and frequency of the wall oscillation are $U_{w}$ and $\Omega$. The parameters governing this modified flow are then $U_{w}$ and $\Omega$, and the Reynolds number $R_{e}$ defined as $h U_{\max } v^{-1}$, where $U_{\max }$ is the maximum velocity of the mean flow in the absence of wall oscillation, $v$ is the kinematic viscosity. The schematic view of the flow field is given in Figure 1.

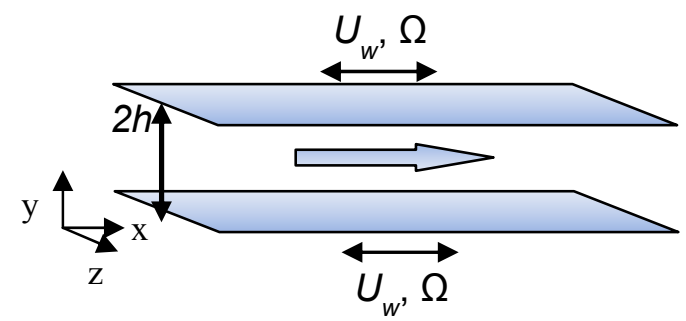

Figure 1: The schematic view of the flow field.

This flow might be an exact solution because the superimposed flow are also the exact solution of the linear equation derived from the Navier-Stokes equation. The mean flow is given as Equation (1).

$$
U(y, t)=1-y^{2}+U_{w} \operatorname{Re}\left[\frac{\cosh (k y)}{\cosh (k)}\right] \exp (i \Omega t) .
$$

Here $k \equiv \sqrt{\Omega / 2 v}, i$ denotes imaginary unit. 


\section{Linear stability analysis}

In the present study, small disturbance $\boldsymbol{u}$ ' existing in the mean flow is assumed as Equation (2).

$$
\boldsymbol{u}^{\prime}(x, y, z, t)=\boldsymbol{u}^{\hat{(}}(y, t) \exp [i(\alpha x \gamma+z)],
$$

here $\alpha$ and $\gamma$ are the wavenumber in $x, z$ direction, respectively. The timedependent N-S equation is obtained by substituting Equation (2) into the linearized disturbance equation, and shown as follows.

$$
\begin{aligned}
& {\left[\left(\frac{\partial}{\partial t}+i \alpha U(y, t)\right)\left(D^{2}-\alpha^{2}-\gamma^{2}\right)-i \alpha D^{2} U(y, t)\right] \hat{v}(y, t)} \\
& =\frac{1}{R}\left(D^{2}-\alpha^{2}-\gamma^{2}\right)^{2} \hat{v}(y, t) .
\end{aligned}
$$

With expansion in the $y$ direction using the Chebyshev spectral collocation method, the above equation can be written as

$$
\left(D_{i j}{ }^{(2)}-\alpha^{2}-\gamma^{2}\right) \frac{d}{d t}\left(\begin{array}{c}
\hat{V}\left(y_{0}, t\right) \\
\hat{V}\left(y_{1}, t\right) \\
\vdots \\
\hat{V}\left(y_{N}, t\right)
\end{array}\right)=g_{i j}\left(\begin{array}{c}
\hat{V}\left(y_{0}, t\right) \\
\hat{V}\left(y_{1}, t\right) \\
\vdots \\
\hat{V}\left(y_{N}, t\right)
\end{array}\right),
$$

here $D_{i j}{ }^{(2)}$ is the differential matrix and the collocation points $y_{i}$ is

$$
y_{j}=\cos \frac{\pi j}{N+1},(j=0,1,2, \cdots, N) .
$$

Since Equation (4) can be rewritten as

$$
\frac{d}{d t} F(t)=G(t) F(t),
$$

the system is described by the Floquet exponents

$$
Q=\frac{1}{T} \ln F .
$$

When the eigenvalue $\mu$ of the matrix $Q$ is positive, the system is unstable.

\section{DNS analysis}

In the present study, the resolution is $64 \times 128 \times 129$ for $4 \pi \times 2 \times 2 \pi$ numerical space. The flow filed consists of the mean flow $U(y, t)$ and the small disturbance $\boldsymbol{u}=\boldsymbol{u}(u, v, w)$. Then $\boldsymbol{u}$ is calculated the following equation 


$$
\frac{\partial \boldsymbol{u}}{\partial t}+U \frac{\partial \boldsymbol{u}}{\partial x}+v \frac{\partial U}{\partial y} \boldsymbol{e}_{\boldsymbol{x}}=-\nabla \times \boldsymbol{u} \times \boldsymbol{u}-\nabla p+\frac{1}{R_{e}} \nabla^{2} \boldsymbol{u},
$$

with the incompressible condition of

$$
\nabla \cdot \boldsymbol{u}=0
$$

Here $\boldsymbol{e}_{x}$ denotes the unit vector in $x$ direction. The velocity $\boldsymbol{u}$ is expanded by the Fourier series for $x$ and $z$ direction, on the Chebyshev collocation points of $y_{i}$.

$$
\boldsymbol{u}(x, y, z, t)=\sum \widetilde{\boldsymbol{u}}(\alpha, y, \gamma, t) \exp [i(\alpha x+\gamma z)]
$$

Thus, energy norm for the Fourier modes per unit mass is defined as follows.

$$
E(\alpha, \gamma, t)=\frac{1}{4} \int_{-1}^{1}|\tilde{\boldsymbol{u}}(\alpha, y, \gamma, t)|^{2} d y .
$$

\section{Results}

\subsection{Linear stability analysis}

For convenience, Reynolds number is fixed to 10,000, hereafter. Under the present definition of Reynolds number, the critical value is about 5,772 for nonoscillating plane Poiseuille flow.

The eigenvalue of Floquet exponent is obtained from Equation (7). The matrix $F$ is numerically integrated Equation 6 during the period $T$. For this integrated process, the Cranc-Nicorson method [13] is employed.

First, the Floquet exponent for the case of $(\alpha, \gamma)=(1.0 .0 .0)$ without oscillation is checked by the comparison with Orszag [1]. Table 1 shows good agreement.

Table 1: Comparison of the Floquet exponent for the case of $\mathrm{Re}=10,000$, $(\alpha, \gamma)=(1.0,0.0)$.

\begin{tabular}{l|l}
\hline Floquet exponent $\mu$ (present) & $0.37397 \mathrm{e}-02$ \\
\hline Eigenvalue $\omega_{i}($ Orszag [1]) & $0.37396 \mathrm{e}-02$ \\
\hline
\end{tabular}

Then, the Floquet exponent is calculated for various set of $\left(U_{w}, \Omega\right)$. Using these results, the contour of the Floquet exponent for the case of $(\alpha, \gamma)=(1.0$. 0.0 ) is shown Figure 2. It can be seen that the stability area exists even though the Reynolds number exceeds the critical value. 


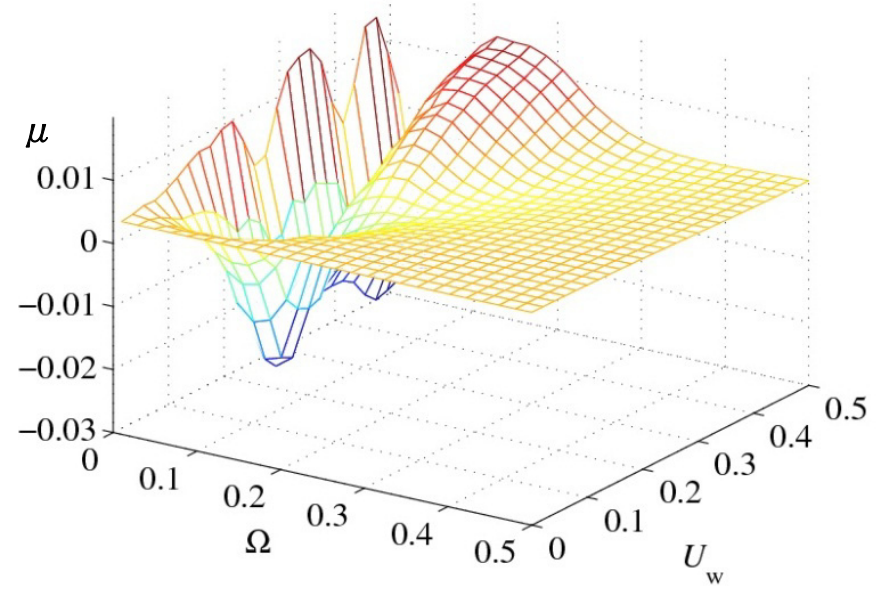

Figure 2: Contour of the Floquet exponent for the case of $(\alpha, \gamma)=(1.0 .0 .0)$.

\subsection{DNS analysis}

Figure 3 shows the variation of the energy for each Fourier mode for the case of $\left(U_{w}, \Omega\right)=(0.0$. 0.0), namely non-oscillating case. At $t=0$, the level of initial disturbances is quite small, and then each Fourier mode grows with $t$. It is found

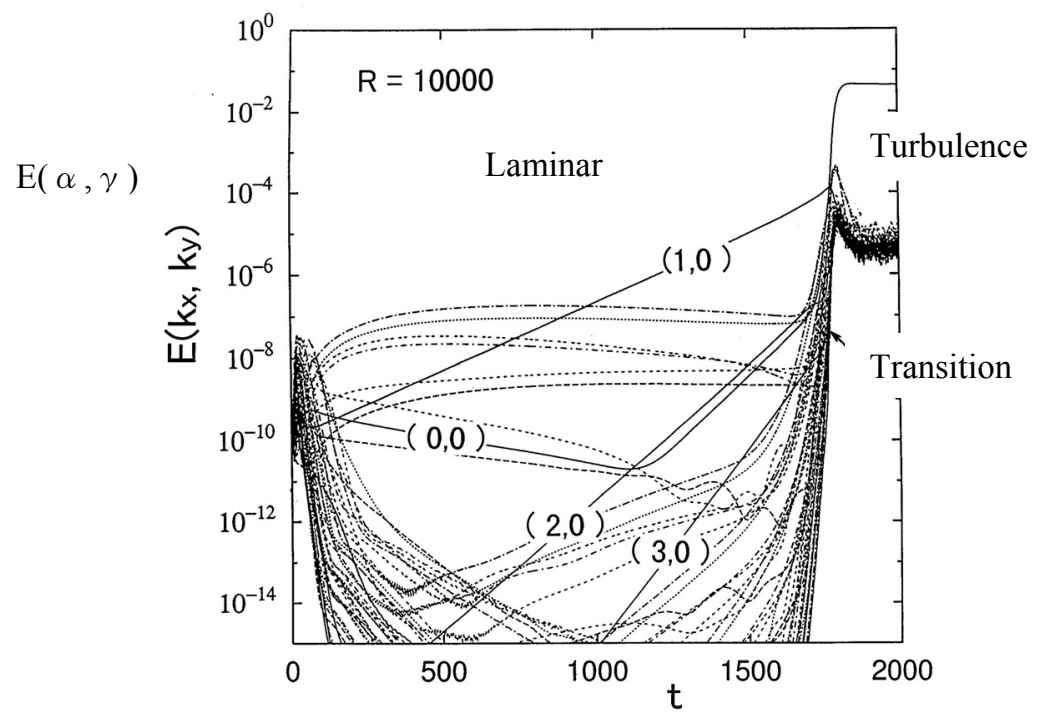

Figure 3: Variation of energy for each Fourier modes for the case of without oscillation. 
that the Tollmein-Schlighting (TS) wave, $(\alpha, \gamma)=(1.0 .0 .0)$, is dominant and finally the flow transits to the turbulence.

Hereafter, TS mode is added as an initial disturbance in order to save the calculation cost. The few examples of the numerical results are shown in Figure 4. Figure 4 (a) corresponds to non-oscillating case. Comparing with the nonoscillating case, it can be seen that there are accelerated, changeless, decelerated cases depending on the parameters. The wavy structure agrees with the period of the wall-oscillation.

Roughly speaking, the feature mentioned above is agrees with the result obtained from the Floquet analysis summarized in Figure 2. These results suggest that the transition phenomena can be inferred from the behaviour of the TS wave.

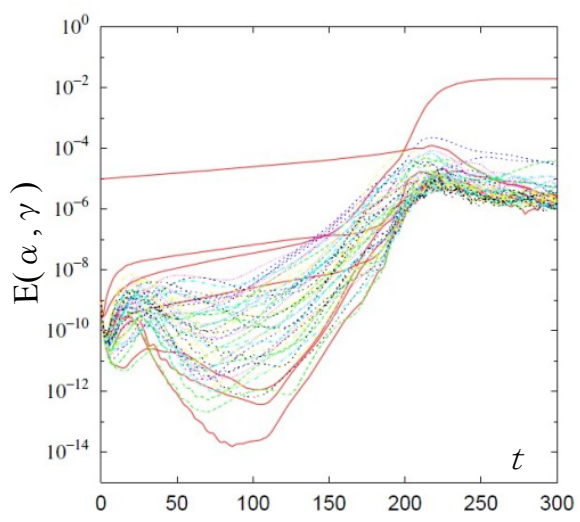

(a) $\left(U_{w}, \Omega\right)=(0.0 .0 .0)$

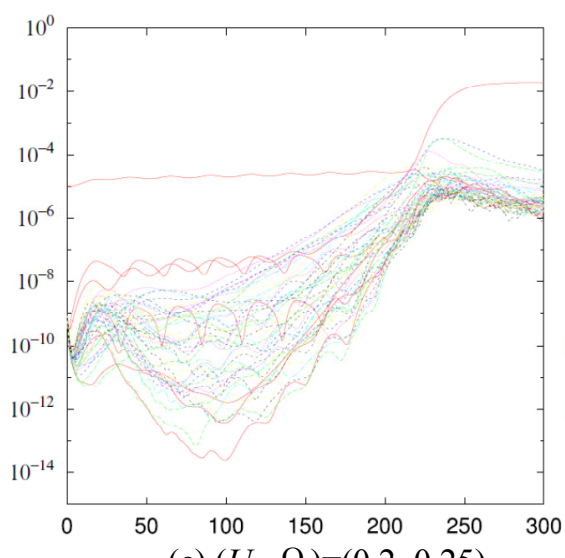

(c) $\left(U_{w}, \Omega\right)=(0.2 .0 .25)$

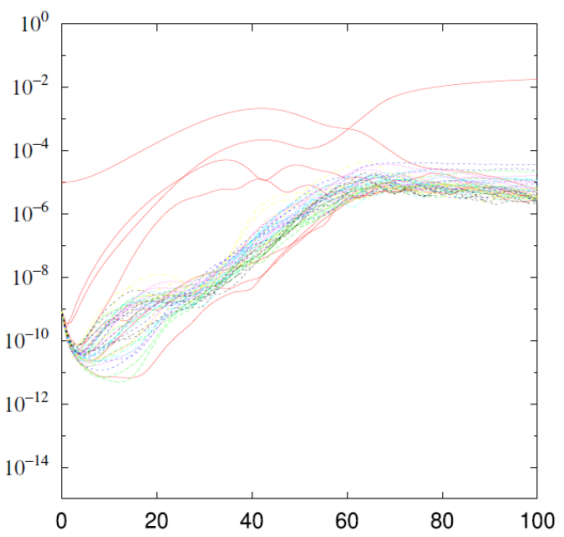

(b) $\left(U_{w}, \Omega\right)=(0.3 \cdot 0.05)$

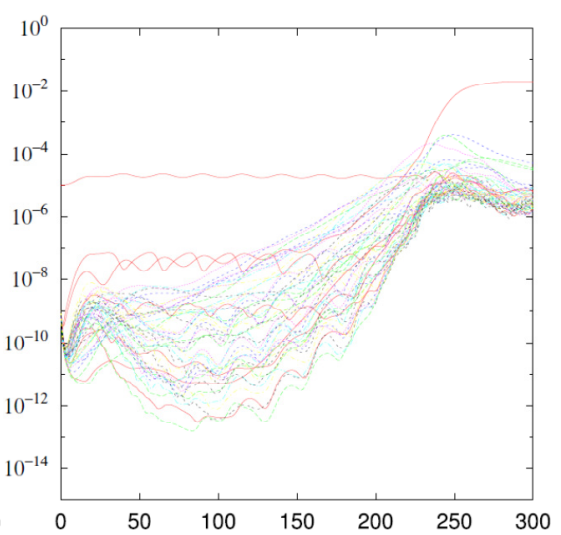

(d) $\left(U_{w}, \Omega\right)=(0.3 .0 .15)$

Figure 4: Variation of energy for each Fourier modes for the case of (a) $\left(U_{w}, \Omega\right)=(0.0 .0 .0),($ b) $(0.3 .0 .05)$, (c) $(0.2 .0 .25),(d)(0.3 .0 .15)$. 
Figure 5 shows the contour of the vorticity just before the transition for the case of non-oscillating case. It can be seen that the flow is complicated though the Lambda-like structure exists at near the wall. On the other hand, for the accelerated case, the streamwise structures periodically distributed are observed. It is conceivable that there is some correlation between these structures and the rapid transition.

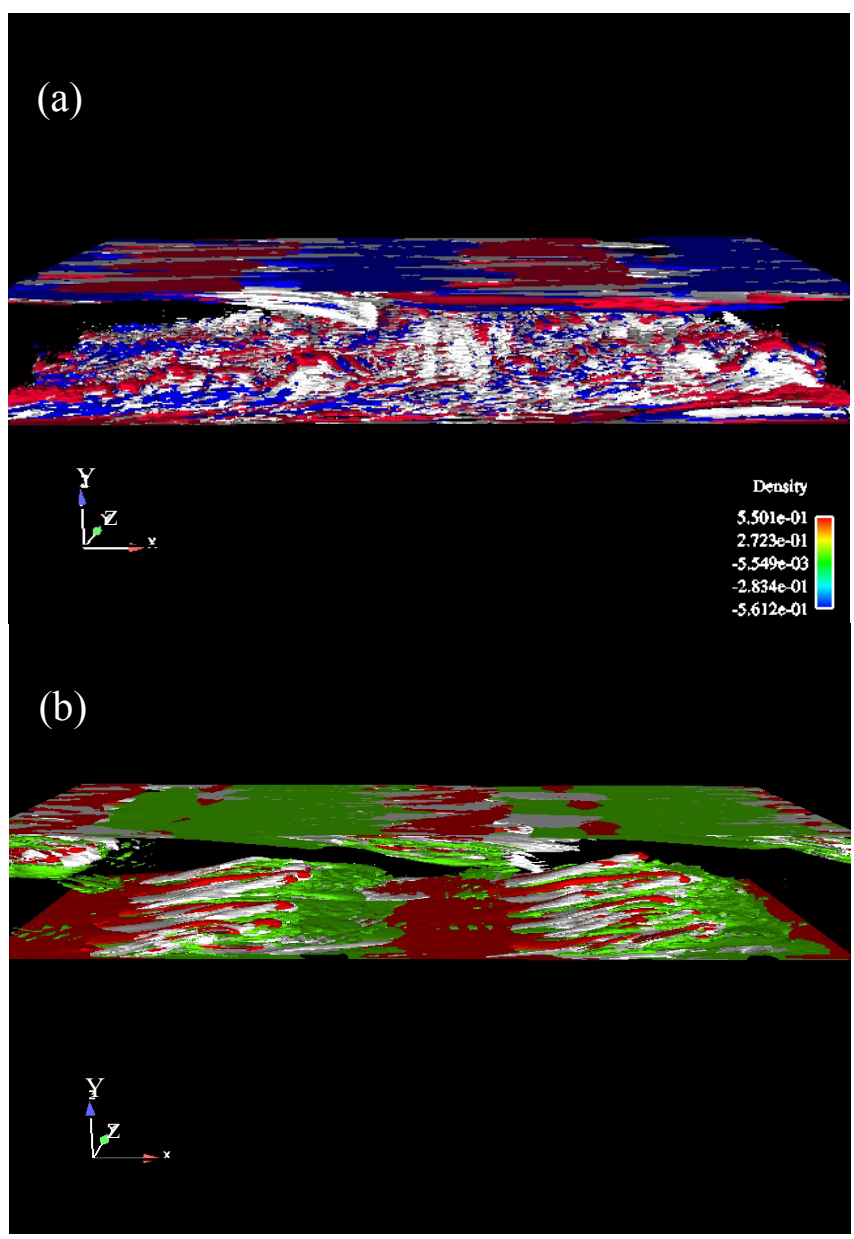

Figure 5: Contour of the vorticity at just before the transition for the case of (a) $\left(U_{w}, \Omega\right)=(0.0 .0 .0)$, (b) $(0.3 .0 .05)$. 


\section{Conclusion}

Influence of wall oscillation on laminar-turbulent transition of the plane Poiseuille flow is investigated analytically and numerically. The Reynolds number defined by the half width of walls and maximum mean velocity is fixed at 10,000 . In the Floquet analysis, a time-dependent Orr-Sommerfeld equation is employed and the velocity profiles are assumed as the superposition of the plane Poiseuille flow and the Stokes layer. The results focused only on TollmeinSchlichtin wave shows existence of the stable region on the parameter space even though under the supercritical condition. Direct numerical simulation also executed using the Fourier spectral method. The results obtained by DNS agree well with the Floquet analysis. These findings imply that the transition phenomenon of the present system is strongly dominated by TS wave. The visualization of the flow field shows a streamwise structure near the wall. This result suggests that there is some correlation between these structures and the rapid transition.

\section{Acknowledgements}

This work was supported by a Grant-in-Aid for Scientific Research (C) from the Japan Society for the Promotion of Science (Grant No. 25420139). Computational resources were provided by JAXA Supercomputer System.

\section{References}

[1] Orszag, S. A.: Accurate Solution of the Orr-Sommerfeld Stability Equation, J. Fluid Mech., 50, 689 (1971), pp. 1441-1447.

[2] Kim, J., Moin, P., and Moser, R..: Turbulence Statistics in Fully Developed Channel Flow at Low Reynolds Number, J. Fluid Mech., 177 (1987), pp. 133-166.

[3] Zamir, M.: The Physics of Pulsatile Flow, Phys. A IP PRESS, Springer (2000).

[4] Jung, W. J., Mangiavacchi, N., and Akhavan, R.: Suppression of Turbulence in Wall-Bounded Flows by High-Frequency Spanwise Oscillations, Phys. Fluids, A 4 (8) (1992), pp. 1605-1607.

[5] Quadrio, M. and Ricco, P.: Critical Assessment of Turbulent Drag Reduction Through Spanwise Wall Oscillation, J. Fluid Mech., 521, (2004), pp. 251-271.

[6] Choi, K.-S.: Near-Wall Structure of Turbulent Boundary Layer with Spanwise-Wall Oscillation, Phys. Fluids, 14 (2002), pp. 2530-2542.

[7] Ricco, P. and Quadrio, M.: Wall-Oscillation Conditions For Drag Reduction in Turbulent Channel Flow, Int. J. Heat Fluid Flow, 29 (4) (2008), pp. 891-902.

[8] Grosch, C. E. and Salwen, H.: The Stability of Steady and Time-dependent Plane Poiseuille Flow, J. Fluid Mech., 34, (1968), pp. 177-205. 
[9] Herbert, D.M..: The Energy Balance in Modulated Plane Poiseuille Flow, J. Fluid Mech., 56, (1972), pp. 73-80.

[10] Hall, P.: The Stability of the Poseuille Flow Modulated at High Frequencies, Proc. R. Soc. Lond. A344, (1975), pp. 453-464.

[11] Davis, S. H.: The Stability of Time-periodic Flows, Annu. Rev. Fluid Mech, (1976), pp. 57-74.

[12] Von Kerczek, C. P.: The Instability of Oscillation Plane Poiseuille Flow, J. Fluid Mech., 116, (1982), pp. 91-114.

[13] Canuto, C., Hussaini, M. Y., Quarteroni, A. and Zang, T. A.: Spectral Methods in Fluid Dynamics, Springer-Verlag, Berlin, 1988. 